\title{
Características reprodutivas de machos bovinos da raça Nelore da fase pré-púbere à maturidade sexual ${ }^{*}$
} Reproductive characteristics of male bovine Nelore breed
of pre pubertal phases to sexual maturity

\author{
Teresinha Inês de Assumpção, ${ }^{* *}$ Marcos de Almeida Souza, ${ }^{* * *}$ Camila Alberton, ${ }^{* * * *}$ Rodrigo Pallaoro, ${ }^{* * * *}$ \\ Clóvis Kitagawa, ${ }^{* * * *}$ Natascha Almeida Marques Silva**
}

\begin{abstract}
Resumo
O objetivo da pesquisa foi analisar as principais características reprodutivas de machos da raça Nelore dos 12 aos 24 meses de idade e as correlações entre estas características. O sêmen foi coletado de 24 animais pela técnica de eletroejaculação, analisado física e morfologicamente e a dosagem de testosterona feita por quimioluminescência. Os dados foram analisados pelos métodos de Pearson e Sperman, análise de variância e teste de Friedman. Os testículos cresceram proporcionalmente com o animal, porém a circunferência escrotal foi baixa em todas as idades sem alterar a produção e qualidade do sêmen. Os animais ganharam 700 gramas/dia de peso e circunferência escrotal aumentou em média 0,037 cm/dia. Aos 17 meses, $50 \%$ dos animais estavam produzindo sêmen, mas com baixa qualidade, se tornando ótima aos 20 meses. A concentração espermática aumentou progressivamente com idade, enquanto houve redução das patologias totais dos espermatozoides. A concentração sérica média de testosterona foi $10,31 \pm 3,15 \mathrm{ng} / \mathrm{mL}$ com grande variação cíclica nas idades. Foi observada uma correlação positiva entre a idade e peso, circunferência escrotal, concentração, motilidade e vigor. Foi observada uma correlação negativa entre a idade e patologias espermáticas. Não houve correlação significativa entre essas características analisadas e a concentração plasmática de testosterona. A circunferência escrotal dos animais aumentou proporcionalmente ao seu desenvolvimento junto com produção e qualidade do sêmen e concentração sérica de testosterona, podendo assim ser utilizada para indicar puberdade. A maturidade sexual dos animais ocorreu em torno dos 20 meses de idade, comprovada pelo espermiograma.
\end{abstract}

Palavras-chave: Bos taurus indicus, circunferência escrotal, reprodução, sêmen, testosterona.

\begin{abstract}
This study aimed to analyze the main reproductive characteristics of Nelore bulls $(n=24)$ from 12 to 24 months old. Semen was collected by electro-ejaculation method and analyzed physically and morphologically. Testosterone was measured by chemiluminescence method. Data were analyzed by Analysis of Variance and Friedman, and their correlations by Pearson and Spearman method. Scrotum circumference increased proportionally to the animal size; although, this circumference was low at all ages without changing production and quality of the semen. Animals gained 700 grams/day in weight; scrotum circumference increased $0.037 \mathrm{~cm} /$ day. Animals presented semen at 17 months with low quality. At 20 m.o. bulls showed good semen quality with spermatozoa concentration increasing according to the age. Age presented negative correlation with semen pathologies. Circulating concentration of testosterone was $10.13 \pm 3.15 \mathrm{ng} / \mathrm{mL}$, with cyclical variation among different ages. There were a positive correlation among age with weight, scrotum circumference, physical characteristics of semen, motility and force. There was a negative correlation between age and sperm pathologies. There was no correlation with any characteristics and testosterone concentration. Scrotal growth of the animals increased proportionally to its development along with production and semen quality and testosterone concentration, which can be used as puberty indicative. Sexual maturity of the animals occurred around 20 months old, confirmed by spermiogram.
\end{abstract}

Keywords: Bos taurus indicus, escrotal circumference, reproduction, semen, testosterone.

\section{Introdução}

A raça Nelore representa em torno de $90 \%$ dos animais das raças zebuínas (Bos taurus indicus) criadas no Brasil, mas ainda apresenta baixos índices de produtividade e reprodutivos $(60 \%$ de taxa de nascimentos). Os machos têm uma alta quota de responsabilidade, já que $95 \%$ das vacas e novilhas do rebanho de corte em condições de reprodução são servidas pela monta natural. Assim, é de grande importância a avaliação do potencial reprodutivo dos reprodutores, principalmente dos machos jovens, com o objetivo de selecionar aqueles que sejam capazes

\footnotetext{
*Recebido em 15 de abril de 2013 e aceito em 13 de agosto de 2013.

**Universidade Federal de Uberlândia, Faculdade de Medicina Veterinária, Uberlândia, MG, Brasil. Autor para correspondência:

E-mail: teassumpcao@famev.ufu.br.

***Universidade Federal de Mato Grosso, Faculdade de Agronomia e Medicina Veterinária, Cuiabá, MT, Brasil.

****Universidade Federal do Mato Grosso, Campus Sinop, Sinop, MT, Brasil.
} 
de imprimir características econômicas desejáveis à sua prole como fertilidade, precocidade, capacidade de ganho rápido de peso e musculosidade (Fonseca, 2009).

A avaliação da fertilidade de um macho é baseada em características do animal e de seu sêmen. O exame clínico do indivíduo, incluindo a medida dos testículos e seu comportamento sexual, aliado ao espermiograma, onde se avaliam as características físicas e as morfológicas do sêmen são a base da seleção reprodutiva (Fonseca et al., 1992; Chenoweth, 2011). A circunferência escrotal (CE) aumenta linearmente com a idade e o peso do animal, aumentando progressivamente com a puberdade, sendo posteriormente mais lento indicando a maturidade sexual (Quirino et al., 1998), e está diretamente ligada à capacidade e o número de espermatozoides produzidos e a reserva espermática (Vásquez et al., 2003). A atividade reprodutiva está diretamente ligada aos testículos que são responsáveis pela espermatogênese e pela produção de andrógenos, principalmente a testosterona que apresenta elevação gradual nas concentrações basais a partir dos 13 meses estando relacionada com a idade e a puberdade (Moura et al., 2002).

A vida reprodutiva do animal se inicia na puberdade com o início da espermatogênese, liberação do pênis, aparecimento da libido e ejaculado com no mínimo 50 milhões de espermatozoides e $10 \%$ de motilidade espermática progressiva (Wolf et al., 1965; Hamilton, 2007). A puberdade sofre a influência de diversos fatores de ambiente e genéticos (Vásquez et al., 2003), apresentando herdabilidade relativamente alta $(0,61)$ (Quirino et al., 1998). Segundo Barth e Ominski (2000) e Rawlings et al. (2008), o método de maior acurácia para predizer puberdade é a CE, pois em animais que possuem testículos pequenos entre 12 e 16 meses, os mesmos continuarão menores por toda a sua vida. A maturidade sexual em bovinos é alcançada com o crescimento gonadal e corporal, com mudança quantitativa e qualitativa do sêmen, e com os níveis de testosterona e desenvolvimento sexual que se estabilizam entre 16 a 20 semanas após a puberdade, onde o animal apresenta sêmen com um mínimo de $50 \%$ de motilidade espermática progressiva e morfologia espermática com no máximo de $10 \%$ de defeitos espermáticos maiores e $20 \%$ de defeitos espermáticos menores (Lunstra e Echternkamp, 1982; Rawlings et al., 2008).

Diante do exposto, este estudo teve por objetivo analisar o desenvolvimento corporal, testicular, parâmetros seminais e a concentração sanguínea de testosterona de machos da raça Nelore dos 12 aos 24 meses de idade, assim como analisar a correlação entre estas características.

\section{Material e métodos}

Foram selecionados 24 machos com idade média de 12 meses, da raça Nelore (Bos taurus indicus), puros de origem, oriundos de uma propriedade localizada na região norte do estado de Mato Grosso (Itaúba /MT). Na região predomina o clima tropical, com temperatura anual variando de $15-40^{\circ} \mathrm{C}$ e pluviometria média de $2.500 \mathrm{~mm}$. Durante toda a pesquisa, os animais foram mantidos em piquetes formado com Tanzânia (Panicum maximum) sob o mesmo regime de manejo e alimentação, mineralização balanceada e suplementação proteico-energética em cocho (no campo) apenas no período da seca ( $2 \mathrm{~kg} /$ cabeça/ dia), mantendo um escore de condição corporal entre 3 e 4 (escala de 1 a 5 ).
Todos os animais foram avaliados por exames clínicos geral e do sistema reprodutivo (Fonseca et al.,1992), sendo a medida da $\mathrm{CE}$, o peso corporal, assim como a coleta de sêmen e sangue foram realizadas quinzenalmente dos 12 aos 21 meses de idade e daí mensalmente até os 24 meses de idade.

A cada análise todos os animais tiveram seus pesos aferidos em balança eletrônica comercial para bovinos (modelo Coimma ${ }^{\circledR}$ ) no início da manhã (animais sem jejum). Os seus testículos foram avaliados quando a simetria (método visual) e a CE avaliada com uma fita métrica flexível. A coleta do sêmen foi feita pelo método de eletroejaculação e o sêmen recolhido após o descarte da primeira porção líquida de plasma seminal, segundo o descrito por Fonseca et al. (1992). A preparação de amostras e as análises físicas e morfológicas do sêmen foram feitas de acordo com as normas do Colégio Brasileiro de Reprodução Animal (CBRA) (Fonseca et al.,1992). No campo logo após a coleta, foram avaliadas a motilidade (MOT) e o vigor sob microscopia óptica. No laboratório de Reprodução Animal da Universidade Federal de Uberlândia (Uberlândia/ $M G$ ), avaliou-se a concentração de células (CONC) utilizando a câmara de Neubauer e a morfologia espermática (PAT) pela técnica de preparação em câmara úmida sob microscopia óptica de contraste de fase, sendo contadas 200 células.

Foram retirados, ao acaso, $50 \%$ dos animais para as coletas de sangue, a fim de realizar a dosagem de testosterona (TEST). A coleta foi sempre realizada dos mesmos animais até o fim da pesquisa, e constou de uma única coleta diária, realizada duas horas após a aplicação de GnRH (Acetato de Gonadorelina), sendo utilizada uma dosagem de $1 \mathrm{mg} / \mathrm{kg}$ p.v., segundo protocolo de Mackinnon et al. (1991). O sangue foi coletado em um volume mínimo de $5 \mathrm{~mL}$ e o soro sanguíneo obtido após centrifugação do sangue a $1500 \mathrm{~g} / 10$ minutos e então congelado $\mathrm{a}-20^{\circ} \mathrm{C}$ até as análises que foram realizadas pela técnica de quimioluminescência com kits Rocha ${ }^{\circledR}$, em aparelho Elecsys (sensibilidade do teste $=3 \mathrm{ng} / \mathrm{dL}$ ) e foram procedidas no laboratório TECSA ${ }^{\circledR}$ em Belo Horizonte/MG.

Para a análise estatística, as variáveis quantitativas (idade, peso, CE, CONC, PAT e TEST) foram correlacionadas pelo método de Pearson e as qualitativas (MOT e vigor) pelo método de Sperman. Para avaliar o comportamento entre as diversas idades (coletas) com as variáveis quantitativas foi feita uma análise de variância seguida de um ajuste de regressão. Para observar as diferenças entre as coletas das variáveis qualitativas (MOT e vigor) foi realizado o teste de Friedman.

\section{Resultados e discussão}

Os resultados obtidos nas diversas avaliações do animal e de seu sêmen encontram-se nas Tabelas 1 e 2. Na Tabela 1, estão os valores médios obtidos para peso, circunferência escrotal, motilidade e vigor do sêmen, concentração de espermatozoides, total de patologias espermáticas e dosagem de testosterona, sendo que os respectivos coeficientes de variação foram: $11,03 \%$ para peso, $9,29 \%$ para $\mathrm{CE}, 35,64 \%$ para CONC, $15,82 \%$ para PAT e $31,10 \%$ para TEST. A Tabela 2 mostra os valores medianos para as comparações de motilidade e vigor entre as idades de 18 a 24 meses dos animais, obtidos pelo teste de Friedman. As análises de regressão das diversas idades em relação ao peso, CE, CONC, PAT e TEST são apresentadas nas Figuras 1Aa $1 \mathrm{E}$. 
Tabela 1: Médias de peso, circunferência escrotal, análises físicas e morfológicas do sêmen e dosagens de testosterona dos animais da raça Nelore dos 12 aos 24 meses de idade

\begin{tabular}{|c|c|c|c|c|c|c|c|c|c|}
\hline Coleta & $\begin{array}{c}\text { Idade } \\
\text { (meses) }\end{array}$ & $\begin{array}{l}\text { Peso } \\
(\mathrm{Kg})\end{array}$ & $\begin{array}{l}\mathrm{CE} \\
(\mathrm{cm})\end{array}$ & $\begin{array}{l}N^{\circ} \text { de animais } \\
\text { com sêmen }\end{array}$ & $\begin{array}{c}\text { MOT } \\
(\%)\end{array}$ & $\begin{array}{l}\text { Vigor } \\
(0 \text { a } 5)\end{array}$ & $\begin{array}{c}\text { CONC } \\
\text { (milhões) }\end{array}$ & $\begin{array}{r}\text { PAT } \\
(\%)\end{array}$ & $\begin{array}{c}\text { TEST } \\
\text { (ng/ml) }\end{array}$ \\
\hline 01 & 12,0 & 245,2 & 18,9 & 01 & 10,0 & 1,0 & 2,0 & 45,0 & 7,43 \\
\hline 02 & 13,0 & 259,5 & 20,2 & 01 & 20,0 & 1,0 & 3,0 & 53,0 & 11,07 \\
\hline 03 & 13,5 & 274,9 & 20,7 & 03 & 20,0 & 1,0 & 6,3 & 39,3 & 10,91 \\
\hline 04 & 14,0 & 281,3 & 21,0 & 07 & 20,0 & 1,0 & 7,2 & 44,0 & 10,94 \\
\hline 05 & 14,5 & 287,0 & 21,3 & 07 & 25,7 & 1,0 & 13,8 & 42,8 & 11,34 \\
\hline 06 & 15,0 & 290,4 & 21,9 & 07 & 31,5 & 1,3 & 14,0 & 39,7 & 10,08 \\
\hline 07 & 15,5 & 301,2 & 22,2 & 07 & 37,5 & 1,5 & 16,2 & 37,6 & 8,62 \\
\hline 08 & 16,0 & 302,7 & 22,9 & 10 & 41,0 & 1,5 & 16,5 & 37,7 & 10,35 \\
\hline 09 & 16,5 & 318,0 & 23,7 & 11 & 43,0 & 1,6 & 23,5 & 32,8 & 10,03 \\
\hline 10 & 17,0 & 319,9 & 24,0 & 13 & 42,0 & 1,5 & 25,2 & 32,1 & 10,06 \\
\hline 11 & 17,5 & 323,8 & 24,8 & 15 & 40,0 & 1,3 & 25,9 & 36,9 & 10,53 \\
\hline 12 & 18,0 & 335,4 & 25,0 & 18 & 41,0 & 1,4 & 29,1 & 33,8 & 9,80 \\
\hline 13 & 18,5 & 342,2 & 25,3 & 20 & 43,0 & 1,6 & 37,7 & 38,1 & 9,73 \\
\hline 14 & 19,0 & 348,1 & 26,4 & 22 & 45,0 & 1,6 & 37,9 & 35,3 & 8,72 \\
\hline 15 & 19,5 & 360,5 & 27,2 & 23 & 47,0 & 1,5 & 41,1 & 34,7 & 9,81 \\
\hline 16 & 20,0 & 353,0 & 27,4 & 23 & 54,0 & 1,7 & 48,4 & 31,6 & 10,01 \\
\hline 17 & 20,5 & 364,4 & 28,3 & 23 & 53,0 & 1,8 & 64,3 & 26,0 & 11,54 \\
\hline 18 & 21,0 & 390,1 & 29,0 & 23 & 58,0 & 2,1 & 76,1 & 22,5 & 10,55 \\
\hline 19 & 22,0 & 393,7 & 29,6 & 24 & 58,0 & 2,1 & 88,5 & 20,8 & 11,45 \\
\hline 20 & 23,0 & 412,1 & 30,3 & 24 & 62,0 & 2,6 & 10,5 & 14,2 & 12,90 \\
\hline 21 & 24,0 & 498,8 & 32,4 & 24 & 75,0 & 2,9 & 145,8 & 10,9 & 13,33 \\
\hline
\end{tabular}

Tabela 2: Valores medianos para comparações de motilidade e vigor do sêmen dos animais da raça Nelore dos 18 aos 24 meses, pelo teste de Friedman

\begin{tabular}{ccc}
\hline Idade (meses) & Motilidade (\%) & Vigor (1 a 5) \\
\hline 18,0 & $45^{\mathrm{c}}$ & $1^{\mathrm{b}}$ \\
18,5 & $45^{\mathrm{bc}}$ & $1,5^{\mathrm{b}}$ \\
19,0 & $50^{\mathrm{bc}}$ & $2^{\mathrm{b}}$ \\
19,5 & $50^{\mathrm{bc}}$ & $2^{\mathrm{b}}$ \\
20,0 & $60^{\mathrm{bc}}$ & $2^{\mathrm{b}}$ \\
20,5 & $60^{\mathrm{bc}}$ & $2^{\mathrm{b}}$ \\
21,0 & $60^{\mathrm{b}}$ & $2^{\mathrm{ab}}$ \\
22,0 & $60^{\mathrm{b}}$ & $2^{\mathrm{ab}}$ \\
23,0 & $70^{\mathrm{ab}}$ & $3^{\mathrm{a}}$ \\
24,0 & $80^{\mathrm{a}}$ & $3^{\mathrm{a}}$ \\
\hline
\end{tabular}

** Letras diferentes nas colunas indicam diferença estatística.

As análises de variância foram feitas das idades de 12 aos 24 meses (representada nas figuras como coletas 1 a 21 - vide Tabela 1) em relação às demais características avaliadas nos animais e no sêmen, verificando o comportamento entre as idades. Foram verificadas regressões do tipo cúbica e quadrática como mostram as Figuras $1 \mathrm{~A}$ a $1 \mathrm{E}$.
A Tabela 1 e as Figuras 1A e 1B mostram o crescimento dos animais e de seus testículos, sendo que as análises de regressão mostraram um comportamento do tipo quadrática $\left(R^{2}=92 \%\right.$ e $R^{2}=99 \%$, respectivamente), mostrando um excelente ajuste da equação estimada, onde o valor observado foi muito próximo ao valor estimado pelas equações de regressão encontradas para as diversas idades. Podemos notar a semelhança entre as Figuras $1 \mathrm{~A}$ e 1B, mostrando que a medida do crescimento do animal e de seu peso, cresce proporcionalmente os seus testículos, porém a CE dos animais desta pesquisa, que foram criados a campo, apresentam-se baixas em todas as idades (abaixo da recomendação do CBRA) sem alterar a qualidade do sêmen. Resultados semelhantes foram obtidos por Silveira et al. (2010), Monteiro et al. (2011) e Segui et al. (2011) que observaram que animais da raça Nelore possuem testículos mais alongados que outras raças indianas, apresentando assim CE menor, mas sem interferir na quantidade e qualidade de sêmen.

Os animais nesta pesquisa foram criados livres em pastagem apenas com suplementação proteico-energética no período seco e tiveram um ganho de peso de aproximadamente 700 gramas/dia, atingindo $335 \mathrm{~kg}$ aos 18 meses, valor este superior ao obtido por Freneau et al. (2006), porém inferior ao verificado por Quirino et al.(1998), talvez devido à variação genética, de manejo e nutrição nos diferentes estudos.

A CE teve um crescimento médio de $0,037 \mathrm{~cm}$ diários ao longo dos 12 meses da pesquisa, com CE apresentando maiores 


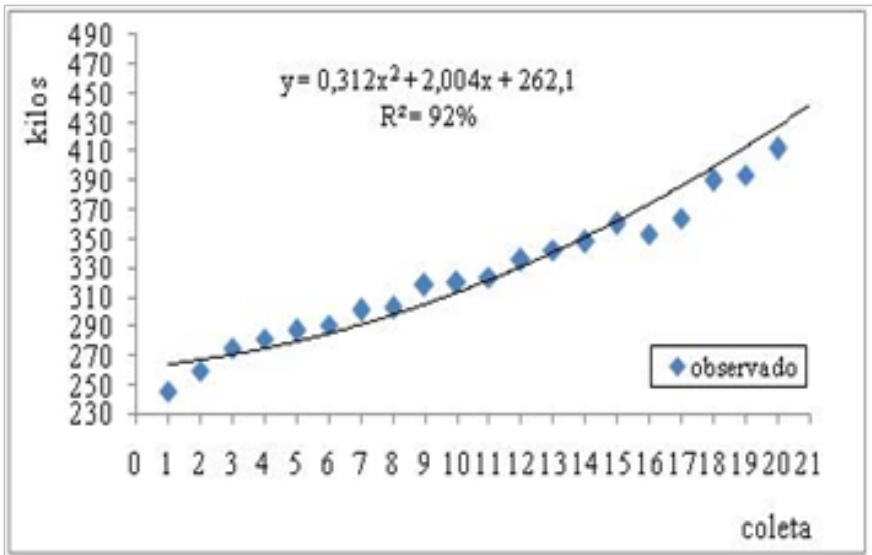

(A)

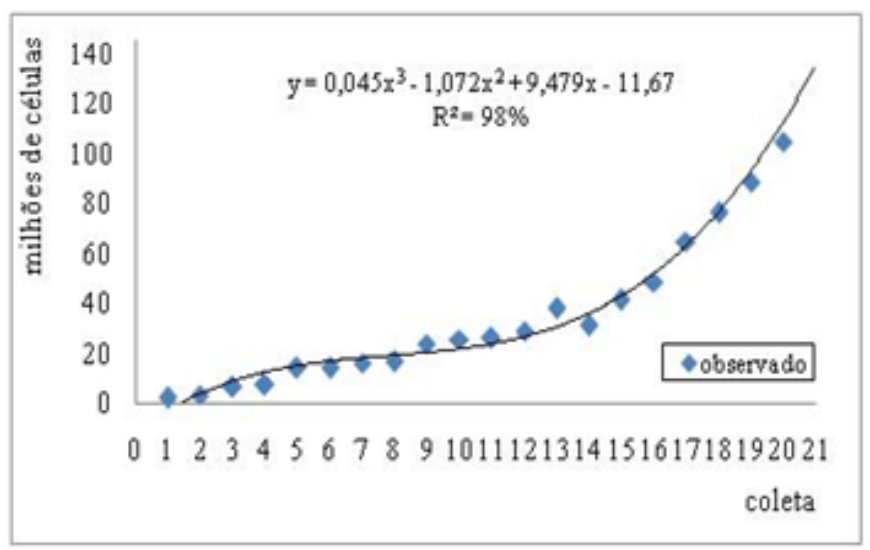

(C)

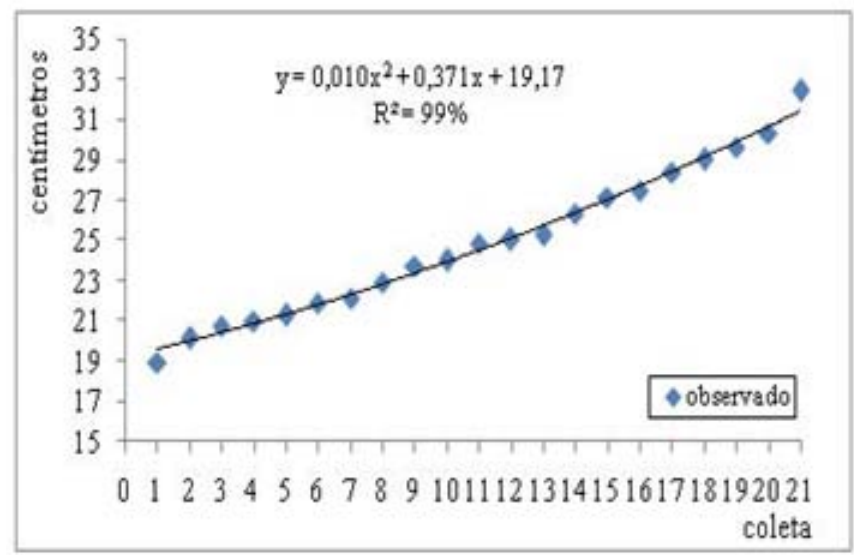

(B)

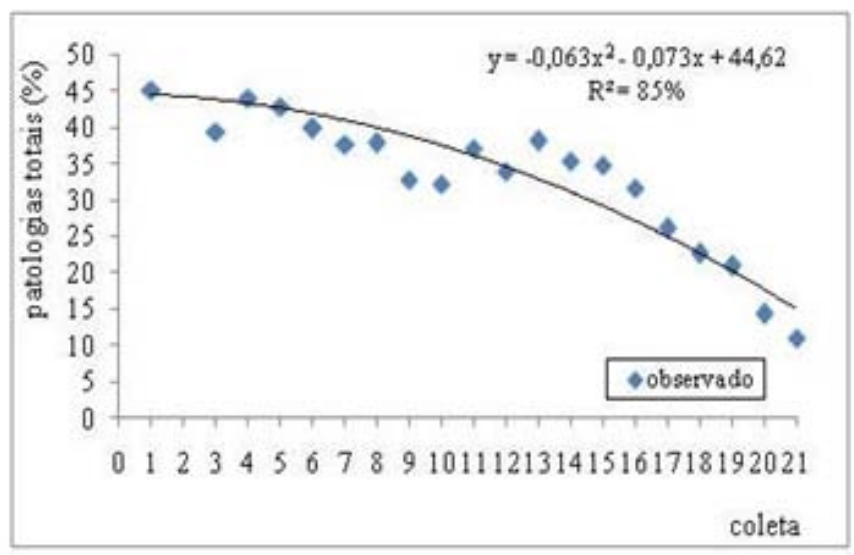

(D)

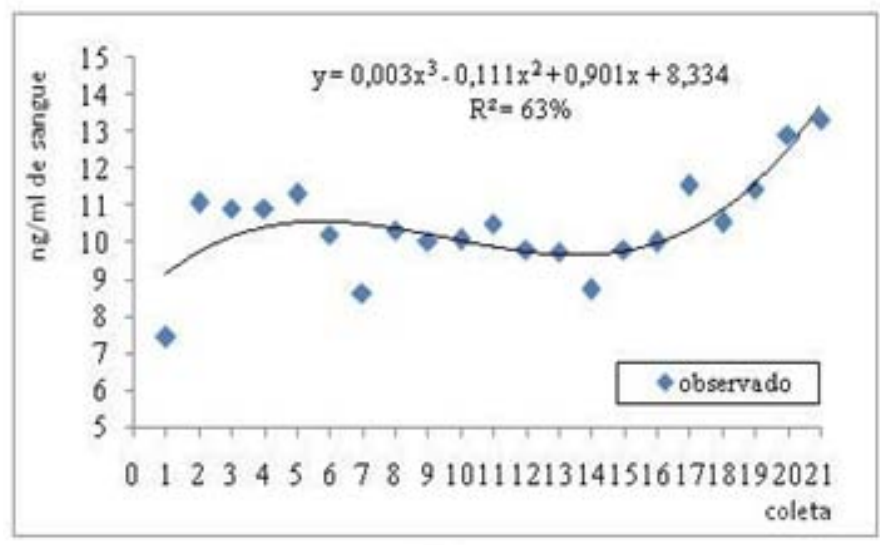

(E)

Figuras 1A a 1E: Análises de regressão das coletas (idades) em relação ao peso (A), circunferência escrotal (B), concentração espermática (C), total de patologias (D) e dosagem de testosterona (E) nos animais da raça Nelore dos 12 aos 24 meses de idade. 
crescimentos entre 12 - 13, 18 - 19 e 23 - 24 meses. Estes valores foram muito superiores aos encontrados por Silva et al. (2002) na mesma raça, que verificaram crescimento testicular de $0,025 \mathrm{~cm} /$ dia até os 18 meses e $0,021 \mathrm{~cm} /$ dia dos 18 aos 24 meses de idade. Também Segui et al. (2011) observaram um maior crescimento testicular dos 10 aos 18 meses $(13,0 \mathrm{~cm})$ com CE atingindo $23,8 \mathrm{~cm}$, tendendo a diminuir a partir daí até os 24 meses $(8,5 \mathrm{~cm})$ com CE chegando a $31,8 \mathrm{~cm}$, assim como Moura et al. (2002) que encontrou um aumento considerável no diâmetro testicular entre 15 e 21 meses. Silva e Tonhati (1997) mostraram que a CE tem crescimento cúbico, ou seja, cresce muito rápido em idades precoces e tende a reduzir seu crescimento com o aumento da idade do animal.

Os animais iniciaram sua produção de sêmen em idades bem variadas, sendo que um deles já apresentava sêmen aos 12 meses (Tabela 1), mostrando sua precocidade, porém, somente após 17 meses o número de animais produzindo sêmen foi superior a $50 \%$, mas ainda com qualidade ruim (baixa MOT e vigor e elevada PAT). A qualidade do sêmen só foi melhorada com o avançar da idade, sendo que a partir dos 20 meses a MOT foi superior a $50 \%$, o vigor próximo a dois e com a PAT abaixo dos $30 \%$, atingindo assim qualidade seminal apropriada para um reprodutor, apesar de sua CE ainda reduzida que só atingiu níveis aceitáveis próximo aos 24 meses. Silva et al. (2002) também verificaram MOT muito baixa até 18 meses (5-20\%), aumentando muito a partir daí, porém com alta variabilidade em testículos do mesmo tamanho, assim como Freneau et al. (2006) que observaram espermatozoides no ejaculado em idades bem precoces (13,6 meses) mas com baixa qualidade (animais Nelore a campo) que só foi melhorada em torno dos 16 meses.

As análises comparativas de MOT e vigor (Tabela 2) foram realizadas apenas a partir dos 18 meses, quando a maioria dos animais já possuía sêmen. Verifica-se que a MOT do sêmen aos 24 meses foi estatisticamente superior aos demais meses e o vigor foi superior aos 23 e 24 meses. Resultado semelhante foi obtido por Monteiro et al. (2011) que observaram bons resultados de MOT $(68 \%)$ e vigor $(3,2)$ os 22,8 meses também em animais da raça Nelore.

Já a CONC mostrou um crescimento progressivo em relação à idade e inversa ao número de PAT do sêmen e na análise de regressão (Figura 1C) mostrou um comportamento do tipo cúbico $\left(R^{2}=98 \%\right)$ mostrando uma grande semelhança entre o encontrado com o estimado estatisticamente pela equação de regressão, o que era esperado devido a maturação testicular. Já a PAT apresentou uma regressão do tipo quadrática (Figura 1D) com $R^{2}=85 \%$, mostrando a tendência de redução nas anormalidades espermáticas com o aumento da idade, resultado semelhante ao observado por Vásquez et al. (2003) em animais da raça Brahman, onde a MOT e a CONC do sêmen teve uma grande elevação dos 15 aos 18 meses, juntamente com a redução das anormalidades dos espermatozoides, sugerindo a maturidade sexual do animal.

A patologia espermática nesta pesquisa só ficou abaixo dos $30 \%$ nos animais acima de 20 meses, semelhante ao verificado por Silva (2007) que observaram a redução de PAT entre 19 e 23 meses nos animais da raça Nelore, porém Vásquez et al. (2003) verificaram expressiva redução na PAT entre 15 aos 18 meses nos animais da raça Brahman.

Em relação à concentração sérica de testosterona, esta teve uma média de $10,31 \pm 3,15 \mathrm{ng} / \mathrm{mL}$ no soro sanguíneo, com o mínimo de 7,43 ng/mL e máximo de 12,43 ng/mL (Coeficiente de variação $=31,10 \%$ ) como mostra a Tabela 1 . A análise de regressão mostrou também um comportamento do tipo cúbico $\left(R^{2}=63 \%\right)$ (Figura 1E) sendo que o ajuste médio da equação é devido a grande variação cíclica na secreção do hormônio verificada nos animais entre as diferentes idades, o que é consequência do desenvolvimento testicular e proliferação das células de Leydig, perfil este também observado por Sanches et al. (1998) e Silva et al. (1999) e Moura et al. (2002). Nesta pesquisa observamos uma variação pequena nos níveis de TEST ao longo do ano, pois o clima na região não apresenta grandes alterações, pois a secreção de TEST pode ser influenciada pelo clima e/ou fotoperíodo segundo Barth e Waldner (2002). As concentrações de testosterona aqui verificadas foram muito mais elevadas que as obtidas por Santos et al. (2000) que encontraram em animais de 24 meses de idade, uma variação de 0,1 a 9,0 ng/mL e os de Sanches et al. (1998) que observaram concentrações de testosterona de $1,95 \mathrm{ng} / \mathrm{mL}$ aos 10 meses, $3,7 \mathrm{ng} / \mathrm{mL}$ aos 12 meses e 5,4 ng/mL aos 15 meses de idade em touros da raça Nelore, ambos dosando testosterona pela técnica de radioimunoensaio. Ainda na mesma raça, também por radioimunoensaio, Silva et al. (1999) registraram concentrações de $0,83 \mathrm{ng} / \mathrm{mL}$ aos 10 meses, $1,73 \mathrm{ng} / \mathrm{mL}$ aos 12 meses e 2,21 ng/ $\mathrm{mL}$ aos 16 meses, valores muito inferiores aos verificados nesta pesquisa. Já a pesquisa de Moura et al. (2002) com animais da raça Nelore de 10 a 30 meses, demonstraram aumento gradual a partir de 13 meses com alta concentração aos 16-18 meses, observando concentrações bem mais elevadas de testosterona nos animais, com variação de 6 a $14 \mathrm{ng} / \mathrm{mL}$, quando a análise foi realizada 3 horas após a aplicação de $\mathrm{GnRH}(0,05 \mathrm{mg} / \mathrm{kg})$, resultados estes semelhantes ao encontrado nesta pesquisa, onde se utilizou também a técnica de pré-aplicação de $\mathrm{GnRH}$. As concentrações séricas de testosterona iguais ou superiores a 1ng/ $\mathrm{mL}$ são consideradas como marcadoras do início da puberdade, uma vez que indica atividade das células de Leydig, responsáveis pela produção de andrógenos, principalmente da testosterona. A concentração de testosterona durante a puberdade está correlacionada com o aumento do número de espermatozoides nos ejaculados e ao decréscimo de anormalidades espermáticas (Evans et al., 1996).

Houve uma grande variação nas idades que os animais iniciaram sua produção de sêmen (Tabela 1), talvez devido à variabilidade genética do grupo, mas em média a puberdade ocorreu entre 15 a 18 meses, semelhante ao observado por Freneau et al. (2006) também em animais da raça Nelore e Trocóniz et al. (1991) que a observaram aos 18,5 meses com CE de 23,6 cm, enquanto que Brito et al. (2004) a verificaram com 20 meses e $C E=24,3 \mathrm{~cm}$. Também Neves (2011) observou a puberdade aos 18 meses com CE maior de $25 \mathrm{~cm}$ semelhante ao verificado nesta pesquisa, afirmando ser esta a idade ideal para selecionar animais jovens para a reprodução.

Em torno dos 20 meses de idade (Tabela 1) os animais tiveram uma elevação da qualidade de seu sêmen, ficando dentro dos parâmetros recomendados pelo CBRA (Fonseca et al., 1992) para animais maduros sexualmente, porém com CE ainda baixa $(27,4 \pm 2,29 \mathrm{~cm})$, sendo que os animais tinham em média pesos superiores a $350 \mathrm{~kg}$. Idades mais tardias de maturidade sexual foram observadas por Silva et al. (2002) aos 24 meses em animais da raça Nelore com $\mathrm{CE}=31,9 \pm 2,6 \mathrm{~cm}$. Monteiro et al. (2011) a verificaram aos 22,8 meses com CE de $32,3 \pm 1,9 \mathrm{~cm}$ com $467 \mathrm{~kg}$ de peso vivo e Brito et al. (2004) que a obtiveram aos 23,8 meses com CE $=29 \mathrm{~cm}$ e $350 \mathrm{~kg}$ de peso. 
As análises de correlações entre as diversas características (idade, peso, CE, CONC, PAT, TEST, MOT e vigor) foram realizadas pelos métodos de correlações de Pearson e Sperman e seus resultados podem ser verificados nas Tabelas 3 e 4, respectivamente.

Tabela 3: Correlações de Pearson verificadas entre as diversas características reprodutivas analisadas nos animais da raça Nelore dos 12 aos 24 meses de idade

\begin{tabular}{lccccc}
\hline & PESO & CE & CONC & PAT & TEST \\
\hline IDADE & 0.7953 & 0.8169 & 0.6088 & -0.4972 & 0.2071 \\
PESO & - & 0.7803 & 0.4893 & -0.4224 & 0.1593 \\
CE & - & - & 0.6022 & -0.5060 & 0.2534 \\
CONC & - & - & - & 0.6309 & -0.0420 \\
PAT & - & - & - & - & 0.1076 \\
\hline
\end{tabular}

CE - circunferência escrotal, CONC - concentração, PAT - patologias totais, TEST - dosagem de testosterona

Tabela 4: Correlações de Sperman entre motilidade e vigor com as demais características reprodutivas analisadas nos animais da raça Nelore dos 12 aos 24 meses de idade

\begin{tabular}{cccccccc}
\hline & IDADE & PESO & CE & CONC & PAT & TEST & MOT \\
\hline MOT & 0.6240 & 0.5439 & 0.6190 & 0.8343 & -0.6983 & 0.0756 & - \\
VIGOR & 0.6140 & 0.5284 & 0.5618 & 0.7246 & -0.6392 & 0.0389 & 0.8313 \\
\hline
\end{tabular}

CE - circunferência escrotal, MOT - motilidade, CONC - concentração, PAT - patologias totais, TEST - dosagem de testosterona

Foram verificadas correlações altas entre idade $x$ peso e idade $x \mathrm{CE}$, assim como entre peso $x \mathrm{CE}$. Já as correlações entre idade, peso e CE $x$ CONC foram médias. Correlações negativas médias foram verificadas entre idade, peso, CE e CONC $x$ PAT, com correlações ausentes ou muito fracas destas características com a dosagem de testosterona do animal. Resultados de correlações próximos a estes foram observados por Silva e Tonhati (1997), Silva et al. (2002), Freneau et al. (2006), Fonseca (2009), Neves (2011) e Segui et al. (2011). Também Moura et al. (2002) verificaram correlação entre idade $x$ CE em todas as idades nos animais da raça Nelore dos 10 aos 30 meses $(r=$ $0,41$ a 0,70$)$.

As análises de correlações das características qualitativas mostraram-se médias em relação a idade, peso e CE, foram altas para CONC e negativas (média a alta) para PAT, não sendo observadas para a concentração de testosterona. Foi observada alta correlação $(r=0,83)$ entre motilidade e vigor, resultado semelhante ao obtido também em animais da raça Nelore por Trocóniz et al.(1991), Freneau et al. (2006), Silva (2007) e Fonseca (2009), assim como Vásquez et al. (2003) na raça Brahman. Silva et al. (2002) também observaram correlação entre a CE e MOT até os 18 meses $(r=0,60)$, não mais a observando a partir desta idade.

O resultado das análises de correlações entre as características analisadas $x$ concentrações séricas de testosterona não foram significativas ou esta foi muito fraca, como mostra a Tabela 3. A correlação aqui observada da CE $x$ TEST foi muito fraca $(r=0,25)$ mas esperada, pois à medida que cresce o animal $\mathrm{e}$ seus testículos, a tendência é crescer também sua produção hormonal, o que foi também observado por Mackinnon et al. (1991). Esta observação foi semelhante à de Brito et al. (2007), que verificaram uma associação entre crescimento do testículo e produção de testosterona, sendo que esta poderia ser usada para indicar o desenvolvimento sexual e a função reprodutiva do animal. Wildeus et al. (1984) observaram resultado semelhante com correlação fraca $(r=0,44)$ entre circunferência escrotal e testosterona em animais mestiços Brahman $x$ Sahiwal, entre 12 e 24 meses de idade, diferente de Chacur et al. (2007) que não obtiveram correlação entre testosterona com as características do sêmen e CE em animais das raças Brangus e Pardo-Suíça.

\section{Conclusões}

A circunferência escrotal em animais da raça Nelore criados a campo é menor que as recomendadas para as diversas idades, porém não há interferência na produção e qualidade de sêmen, podendo assim ser utilizada para indicar puberdade e selecionar reprodutores jovens.

As concentrações de testosterona apresentam grande variação nos animais, com um perfil cíclico e valores bem elevados para todas as idades.

As características físicas do sêmen (motilidade, vigor e concentração) tendem a se elevar com a idade do animal, inversamente ao que ocorre com a patologia espermática e tem correlação positiva com a circunferência escrotal, idade e peso do animal.

A maturidade sexual dos animais ocorreu em torno dos 20 meses de idade, comprovada pelo espermiograma.

\section{Agradecimentos}

À Fundação de Amparo a Pesquisa do Estado de Mato Grosso pelo financiamento da pesquisa. À Fazenda Macuco (Itaúba/MT) pela concessão dos animais. À Universidade Federal do Mato Grosso/ Campus Sinop pelo apoio logístico para realização do projeto.

\section{Referências}

BARTH, A.D.; WALDNER, C.L. Factors affecting breeding soundness classification of beef bulls in Saskatchewan. Canadian Veterinary journal, v. 43, p. 274-284, 2002.

BARTH, A.D.; OMININSKI, K.H. The relationship between scrotal circumference ao weaning and at one year of age in beef bulls. Canadian Veterinary Journal, v. 4, p. 541-546, 2000.
BERGMANN, J.A.G. Melhoramento genético da eficiência reprodutiva em bovinos de corte. Revista Brasileira de Reprodução Animal, n.10, p. 70-86, 1993.

BRITO, L.F.C.; SILVA, A.E.D.F.; UNANIAN, M.M.; DODE, M.A.N.; BARBOSA, R.T.; KASTELIC, J.P. Sexual development in early and late-maturing Bos indicus and Bos indicus $x$ Bos taurus crossbred bulls in Brazil. Theriogenology, v. 62, n. 7, p. 1198-1217, 2004. 
BRITO, L.F.C.; BARTH, A.D.; RAWLINGS, N.C.; WILDE, R.E.; Crews Jr., D.H.; MIR, P.S.; KASTELIC, J.P. Circulating metabolic hormones during the peripubertal period and their association with testicular development in bulls. Domestic Animal Endocrinology, v. 42, p. 502-508, 2007.

CHACUR, M.G.M.; SIRCHIA, F.P.; ZERBINATTI, E.P.; KRONKA, S.N.; OBA, E. Relação entre circunferência escrotal, libido, hormônios e características do sêmen em touros Brangus e PardoSuíço. Acta Scientiae Veterinariae, v. 35, p.173-179, 2007.

CHENOWETH, P.J. Reproductive selection of males: current and future perspectives. Revista Brasileira Reprodução Animal, v. 35, n. 2, p. 133-138, 2011.

EVANS A.C.O.; PIERSON, R.A.; GARCIA, A.; MCDOUGALL, L.M.; RAWLINGS, N.C. Chances in circulating hormone concentrations, test histology and testes ultrasonography during sexual maturation in beef bull. Theriogenology, v. 31, n. 2, p. 345-357, 1996.

FONSECA, V.O. Avaliação reprodutiva de touros para a monta a campo: análise crítica. Revista Brasileira Reprodução Animal, supl.6, p. 36-41, 2009.

FONSECA, V.O., VALE FILHO, V.R., MIES FILHO, A. ABREU, J.J. Procedimentos para exame andrológico e avaliação de sêmen animal, Belo Horizonte: Colégio Brasileiro de Reprodução Animal, 1992, $79 \mathrm{p}$.

FRENEAU, G.E.; VALE FILHO, V.R.; MARQUES JUNIOR, A.P.; MARIA, W.S. Puberdade em touros Nelore criados em pasto no Brasil: características corporais, testiculares e seminais e de índice de capacidade andrológica por pontos. Arquivo Brasileiro de Medicina Veterinária e Zootecnia, v. 58, p. 1107-1115, 2006.

HAMILTON, T. Maximizing Beef Bull Fertility and Reproduction, 2007. Disponível em: http://www.thecattlesite.com/ Acessado em: 22/02/2013.

LUNSTRA, D.D., ECHTERNKAMP, S.E. Puberty in beef bulls: acrosome morphology and semen quality in bulls of different breeds. Journal Animal Science, v. 55, n. 3, p. 638-648, 1982.

MACKINNON, M.J., CORBET, N.J., BURROW, H.M.; BRYAN, R.P.; HETZEL,D.J.S. Genetic parameters for testosterone response to GNRH stimulation and scrotal circunference in tropical beef bulls. Livestock Production Science, v. 29, p. 297-309, 1991.

MONTEIRO, F.M.; OLIVEIRA, L.Z.; OLIVEIRA, C.S.; TETZNER, T.A.D.; MERCADANTE, M.E.Z.; BRANCO, R.H.; GARCIA, J.M. Avaliação andrológica de touros jovens de diferentes raças selecionados para peso pós-desmama. Boletim de Indústria Animal, v. 68, p. 37-43, 2011.

MOURA, A.A.A.; RODRIGUES, G.C.; MARTINS FILHO, O.R. Desenvolvimento Ponderal e testicular, concentrações periféricas de testosterona e características de abate em touros da raça Nelore. Revista Brasileira de Zootecnia, v. 31, n. 2, p. 3107-3115, 2002.

NEVES, A.L.A.; DEL REI, A.J.; SANTOS, M.P. Crescimento testicular de touros da raça Nelore. Livestock Research for Rural Development, v. 23, p. 93-100, 2011.

QUIRINO, C.; BERGMANN, J.; BERGMANN, J.A.G. Heritability of scrotal circumference adjusted and unadjusted for body weight in nellore bulls, using univariate and bivariate animal models. Theriogenology, v. 49, n. 7, p. 1389-1396, 1998.
RAWLINGS, N.; EVANS A. C.O.; CHANDOLIA, R.K.; BAGU, E.T. Sexual maturation in the bull. Reproduction Domestic Animals, v.43, suppl.2, p. 295-301, 2008.

RODRIGUEZ-MARTINEZ, H.; BARTH, A.D. In vitro evaluation of sperm quality related to in vivo function and fertility. Society of Reproduction and Fertility, Suppl. 64, p. 39-54, 2007.

SANCHES, A.C.; LÔBO, R.B.; BEZERRA, L.A.F. Efeito do manejo nutricional sobre medidas lineares e níveis de testosterona em touros zebuínos jovens. Revista Brasileira de Zootecnia, n. 35, p. 46, 1998.

SANTOS, D.M., TORRES, A.C.A.; MENDES RUAS, J.R.; MACHADO, G.V.; COSTA, D.S.; ANGULO, L.M. Concentração sérica de testosterona em touros Zebu. Revista Brasileira de Zootecnia, v. 39, n. 3, p. 738-744, 2000.

SEGUI, M.S.; TURRA, T.A.; FALEIROS, E.; WEISS, R.R.; KOSICKI, L.E. ; SANTOS, I.W. Correlação entre biometria testicular, a idade e as características reprodutivas de touros da raça Nelore. Archives of Veterinary Science, v. 16, n. 1, p. 1-6, 2011.

SILVA, A.S.F. Maturidade sexual e congelabilidade do sêmen de tourinhos Gir-L, sob manejo alimentar estratégico, 2007. 42 p. Dissertação (Mestrado em Medicina Veterinária) - Universidade Federal de Minas Gerais, Belo Horizonte, 2007.

SILVA, J.A.V.; TONHATI, H. Estudo do perímetro escrotal e peso corporal de um rebanho da raça Nelore. In: REUNIÃO ANUAL DA SOCIEDADE BRASILEIRA DE ZOOTECNIA, XXIV. 1997, Anais..., Juiz de Fora: SBZ, 1997, p. 257-259.

SILVA, A.E.D.F.; UNANIAN, M.M.; CORDEIRO, C.M.T.; FREITAS, A.R. Relação da circunferência escrotal e parâmetros de qualidade de sêmen em touros da raça nelore PO. Revista Brasileira de Zootecnia, v. 31, n. 3, p.1157-1165, 2002.

SILVA, A.E.D.F.; UNANIAN, M.M. ; SILVA, A.R. Characterization of sexual precocity in purebred Nelore bulls. Brazilian Archives of Biology and Technology, v. 42, n. 4, p. 495-500, 1999.

SILVEIRA, T.S.; SIQUEIRA, J.B.; GUIMARÃES, S.E.F.; PAULA, T.A.R.; NETO, T.M; GUIMARÃES, J.D. Maturação sexual e parâmetros reprodutivos em touros da raça nelore criados em sistema extensivo. Revista Brasileira de Zootecnia, v. 39, n. 3, p. 36-39, 2010.

TROCÓNIZ, J.F.; BÉLTRAN, J.; BASTIDAS,H.; LARREAL, H.; BASTIDAS, P. Testicular development, body weight changes, puberty and semen traits of growing Guzerat and Nellore bulls. Theriogenology, v. 35, n. 4, p. 815-826, 1991.

VÁSQUEZ, L.; VERA, O.; ARANGO, J. Testicular growth and semen quality in peripuberty Brahman bulls. Livestock Research for Rural Development, v.15, n. 10, 2003.

WILDEUS, S.; HOLROYD, R.G., ENTWISTLE, K.W. Patterns of puberal development in Sahiwal and Brahman cross bulls in tropical Australia. I. Growth and semen characteristics. Theriogenology, p. 22, p. 361-373, 1984.

WOLF, F.R.; ALMQUIST, J.O.; HALE, E.B. Pubertal behaviour and pubertal characteristics of beef bulls on high nutrition allowance. Journal of Animal Science, n. 24, p. 761-765, 1965. 